\title{
miR-374 promotes myocardial hypertrophy by negatively regulating vascular endothelial growth factor receptor-1 signaling
}

\author{
Jong Sub Lee, Dong Woo Song, Jei Hyoung Park, Jin Ock Kim, Chunghee Cho \& Do Han Kim ${ }^{*}$ \\ School of Life Sciences and Systems Biology Research Center, Gwangju Institute of Science and Technology (GIST), Gwangju 61005, Korea
}

\begin{abstract}
Vascular endothelial growth factor (VEGF) is an essential cytokine that has functions in the formation of new blood vessels and regression of cardiac hypertrophy. VEGF/VEGFreceptor-1 (VEGFR1) signaling plays a key role in the regression of cardiac hypertrophy, whereas VEGF/VEGFR2 signaling leads to cardiac hypertrophy. In this study, we identified the prohypertrophic role of $m i R-374$ using neonatal rat ventricular myocytes (NRVMs). Our results showed that overexpression of miR-374 activated G protein-coupled receptor-mediated prohypertrophic pathways by the inhibition of VEGFR1-dependent regression pathways. Luciferase assays revealed that $m i R-374$ could directly target the 3 '-untranslated regions of VEGFR1 and cGMP-dependent protein kinase-1. Collectively, these findings demonstrated that miR-374 was a novel pro-hypertrophic microRNA functioning to suppress the VEGFR1-mediated regression pathway. [BMB Reports 2017; 50(4): 208-213]
\end{abstract}

\section{INTRODUCTION}

Cardiac hypertrophy is defined as the enlargement of the heart accompanied by increased cardiomyocyte size, highly organized sarcomeres, and re-activation of fetal genes. Initially, hypertrophic remodeling serves an adaptive function in response to abnormal overloading. However, in the presence of prolonged pathological stimuli, the remodeling processes can become maladaptive, leading to heart failure (1). The major feature of cardiac remodeling is hypertrophic growth of cardiac myocytes, which is associated with the activation of membrane receptors, such as $\mathrm{G}$ protein-coupled

*Corresponding author. Tel: +82-62-715-2485; Fax: +82-62-7153411; E-mail: dhkim@gist.ac.kr

https://doi.org/10.5483/BMBRep.2017.50.4.165

Received 27 September 2016, Revised 17 October 2016, Accepted 28 October 2016

Keywords: Cardiac hypertrophy, cGMP-dependent protein kinase-1, MicroRNA, Neonatal rat ventricular myocytes, Vascular endothelial growth factor signaling receptors (GPCRs), owing to increased levels of neurohumoral hormones, including norepinephrine (NE) and endothelin-1 (ET-1) (2). These processes also involve downstream signaling molecules such as nuclear factor of activated T-cells (NFAT), phosphoinositide 3-kinase (PI3K), protein kinase C (PKC), and mitogen-activated protein kinases (MAPKs) (3).

Vascular endothelial growth factor (VEGF) is an endothelial cell mitogen that plays an important role in both vasculogenesis and angiogenesis (4). VEGF is also essential in regression of hypertrophied cardiomyocytes (5). VEGF is known to be produced in NRVMs and secreted to the culture media. The biological roles of secreted VEGF are determined by its interaction with two main tyrosine kinase receptors, VEGFR1 and VEGFR2 (6). Binding of VEGF to VEGFR2 can induces hypertrophic growth through activation of the Akt1 (protein kinase B), PKC, and ERK1/2 (extracellular signalregulated kinase 1/2) signaling pathways (7-9). However, in the presence of copper $(\mathrm{Cu}), \mathrm{VEGFR} 2$ is markedly reduced; therefore, VEGF predominately binds to VEGFR1, resulting in the regression of cardiomyocyte hypertrophy through the activation of cGMP-dependent protein kinase-1 (PKG-1) (6).

MicroRNAs (miRNAs) are a small noncoding RNA molecules that suppresses target gene expression by directly binding to the 3'-untranslated regions (3'-UTRs) of the target mRNAs, thereby reducing transcriptional stability and/or translational efficiency (10). A number of recent studies have shown that the regulatory functions of miRNAs in gene expression are involved in cardiovascular diseases (11).

In the previous study involving a cardiac-specific gene set analysis, we identified 18 miRNAs, including miR-374, of which target genes are significantly enriched in cardiac hypertrophy signaling pathways (12). In the present study, we attempted to elucidate the molecular mechanisms through which miR-374 is involved in cardiac hypertrophy. We found that miR-374 inhibited the VEGFR1 signaling pathway by directly targeting VEGFR1 and PKG-1, resulting in activation of GPCR signaling molecules such as NFAT, calcium/calmodulindependent protein kinase II (CaMKII), and myocytes enhancer factor-2 (MEF2).

ISSN: 1976-670X (electronic edition)

Copyright (C) 2017 by the The Korean Society for Biochemistry and Molecular Biology

(c) This is an open-access article distributed under the terms of the Creative Commons Attribution Non-Commercial License (http://creativecommons.org/licenses/by-nc/4.0) which permits unrestricted non-commercial use, distribution, and reproduction in any medium, provided the original work is properly cited. 


\section{RESULTS}

Overexpression of $\mathbf{m i R}-\mathbf{3 7 4}$ induced myocardial hypertrophy To determine the role of miR-374 in the heart, we overexpressed miR-374 in neonatal rat ventricular myocytes (NRVMs). Transfection of a miR-374 mimic (final concentration: $20 \mathrm{nM}$ ) increased cell surface area, compared with that of negative control mimic (NC)-transfected group. In the presence of $10 \mathrm{nM} \mathrm{ET}-1$, miR-374 further increased cell size (Fig. 1A and $\mathrm{B})$. We also performed quantitative real-time reverse transcription polymerase chain reaction (qRT-PCR) to examine the expression levels of hypertrophic markers, such as atrial natriuretic factor (ANF) and brain natriuretic peptide (BNP). We found that miR-374 overexpression upregulated these hypertrophic markers in the presence or absence of ET-1 (Fig. $1 C$ and D). On the other hand, transfection of anti-miR-374

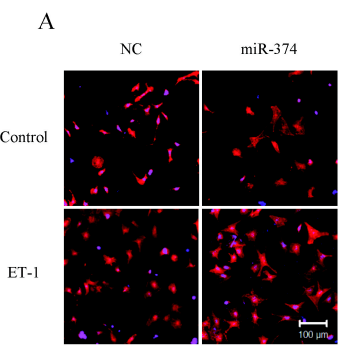

C

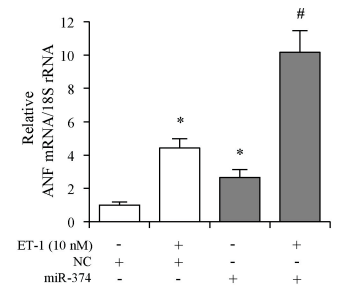

B

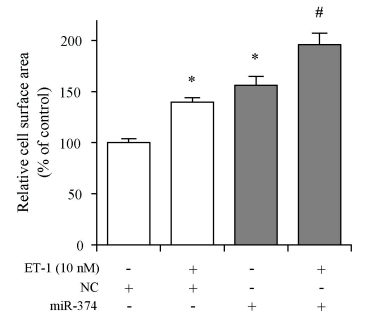

D

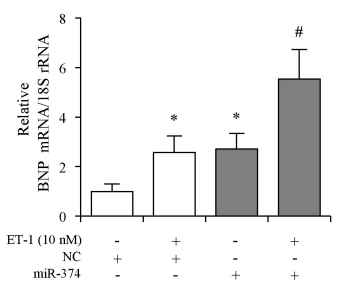

Fig. 1. Overexpression of miR-374 induced cardiomyocyte hypertrophy. (A) Representative photographs of NRVMs transfected with either $20 \mathrm{nM} \mathrm{NC}$ or miR-374 after treatment with $10 \mathrm{nM}$ ET-1 for 24 h. Sarcomeric organization of the cardiomyocytes was visualized by staining with an anti- $\alpha$-actinin antibody. Scale bar, $100 \mu \mathrm{m}$. (B) Cell surface areas shown in Fig. 1A were measured using NIH Imagej software ( $\mathrm{n}=100$ cells per condition). The data show fold changes \pm SDs compared with the control (no agonist, NC added). Significance was measured via two-way ANOVA. *P $<0.05$ compared with the NC control, and ${ }^{\#} \mathrm{P}<0.05$ compared with the miR-374 control. (C, D) Expression of $A N F$ and $B N P$ mRNAs was measured by qRT-PCR in NRVMs in the presence or absence of miR-374 or ET-1. The qRT-PCR analysis was performed in triplicate with three independent samples. Data are expressed as fold changes \pm SD versus the control group. Significance was measured via two-way ANOVA. $* P<0.05$ compared with the $\mathrm{NC}$ control, and ${ }^{\#} \mathrm{P}<0.05$ compared with the miR-374 control. $\mathrm{NC}$, negative control miRNA mimic; miR-374, miR-374 mimic; ET-1, endothelin-1. markedly decreased miR-374 expression resulting in reduced expression of hypertrophic markers in the presence or absence of ET-1 (Fig. S4). Collectively, these findings suggested that miR-374 positively regulated myocardial hypertrophy.

\section{miR-374 directly targets VEGFR1 and PKG-1, the key components responsible for the cardiac hypertrophy regression pathway}

Because miRNAs can have multiple targets involved in the same signaling pathways (13-15), the computational target prediction tool TargetScan (http://targetscan.org) was used to identify putative targets of miR-374. mRNAs encoding VEGFR1 and PKG-1, the key signaling molecules involved in the cardiac hypertrophy regression pathway, were found to contain conserved seed sites for miR-374 in their 3'-UTRs (Fig. 2A and C). To determine whether miR-374 directly targeted VEGFR1 and PKG-1 mRNAs, we performed luciferase assays using cloned 3'-UTRs of the putative target genes. Co-transfection of the reporter plasmid harboring the $3^{\prime}-\mathrm{UTR}$ of VEGFR1 or
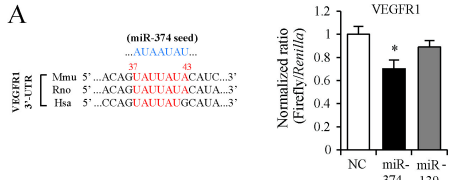

$\mathrm{C}$
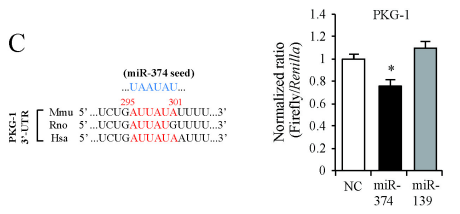
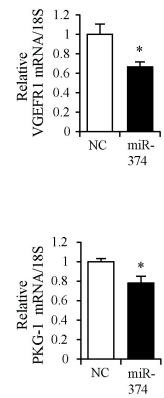

E
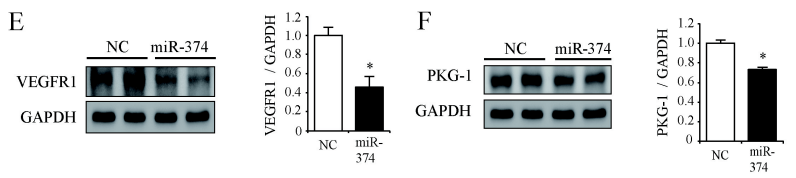

Fig. 2. miR-374 directly targets VEGFR1 and PKG-1 (A, C). Sequence alignments of 3 '-UTRs of mammalian VEGFR1 and PKG-1. miR-374 target region of VEGFR1 and PKG-1 3'-UTRs were well conserved. The dual luciferase reporter vector (pmiRGLO) harboring the VEGFR1 or PKG-1 3'-UTR was cotransfected with NC or miR-374 (20 nM each), into HEK293 cells. The relative firefly luciferase activity was measured and normalized to that of Renilla luciferase. miR-139 did not have a specific seed region for the 3'-UTRs of VEGFR1 and PKG-1, and was therefore used as another negative control. (B, D) qRT-PCR analysis measuring the expression of VEGFR1 and PKG-1 mRNA in NRVMs transfected with either NC or miR-374. (E, F) Western blot analysis measuring VEGFR1 and PKG-1 protein expression in NC or miR-374-transfected NRVMs. The expression levels of the target proteins were estimated by measuring band densities using $\mathrm{NIH}$ Image software. GAPDH was used as a loading control. Data are shown as fold changes \pm SDs versus the control group $(\mathrm{n}=3)$. Significance was measured via two-way ANOVA. $* \mathrm{P}<$ 0.05 compared with the NC control. NC, negative control miRNA mimic; miR-374, miR-374 mimic. 
PKG-1 with NC mimics or miR-374 mimics showed that miR-374 significantly reduced luciferase activity compared with NC or another negative control, miR-139 (Fig. 2A and C).

Next, we performed western blot analysis to examine the expression levels of the two putative targets of $m i R-374$. The results showed that miR-374 significantly decreased the expression levels of VEGFR1 and PKG-1 (54\% and 27\%, respectively) compared with $\mathrm{NC}$ (Fig. 2E and F). mRNA levels of the two target genes were also examined by qRT-PCR. The mRNA levels of VEGFR1 and PKG-1 were also significantly downregulated by miR-374 (Fig. 2B and D). Collectively, these data demonstrated that miR-374 could suppress the expression of VEGFR1 and PKG-1 at both the transcriptional and translational levels.

miR-374-activated myocardial hypertrophy was caused by inhibition of GPCR-associated $\mathrm{Ca}^{2+}$-dependent prohypertrophic pathways through targeting of VEGFR1 and PKG-1

To test the hypothesis that $m i R-374$ positively regulates cardiac hypertrophy through inhibition of VEGFR1 and PKG-1 and subsequent activation of GPCR-associated prohypertrophic $\mathrm{Ca}^{2+}$ signaling (Fig. S1), we performed western blotting to examine the key molecules involved in the downstream signaling pathways, such as CaMKII, NFATc3, and NFATc4. The results showed that CaMKII phosphorylation was increased, whereas NFATc3 and NFATc4 phosphorylation were downregulated in response to miR-374 overexpression, suggesting that miR-374 activated cardiac hypertrophy via activation of the $\mathrm{Ca}^{2+}$-signaling pathways. To confirm these findings, we performed luciferase assays using a $9 \times$ NFAT-luc vector carrying nine tandem-repeat NFAT-binding sites or a $3 \times$ MEF2-luc vector carrying three tandem-repeat MEF2binding sites in the promoter region of firefly luciferase (Fig. 3C-E). As expected, luciferase activity was increased in ET-1-treated groups, compared with that in the control group for both NFAT-Luc (Fig. 3D) and MEF2-Luc (Fig. 3E). Luciferase activities for both NFAT and MEF2 were significantly increased by overexpression of $\mathrm{miR}-374$ in the presence and absence of ET-1 treatment.

Collectively, the present results suggested that miR-374 may play a prohypertrophic role in cardiomyocytes through inhibition of VEGFR1 and PKG-1 and subsequent activation of $\mathrm{Ca}^{2+}$-dependent prohypertrophic signaling molecules.

\section{Inhibition of VEGFR1 induced cardiomyocyte hypertrophy}

The data shown in Figs. 1-3, suggested that targeting of VEGFR1 or PKG-1 by miR-374 induced myocardial hypertrophy through activation of GPCR-mediated prohypertrophic $\mathrm{Ca}^{2+}$ signaling pathways (Fig. S1). However, analysis of the direct effects of inhibition of VEGFR1 or PKG-1 on cardiomyocytes might further support our hypothesis. Transfection of 50 nM VEGFR1 siRNA significantly suppressed the mRNA expression level by $\sim 40 \%$, similar to expression after

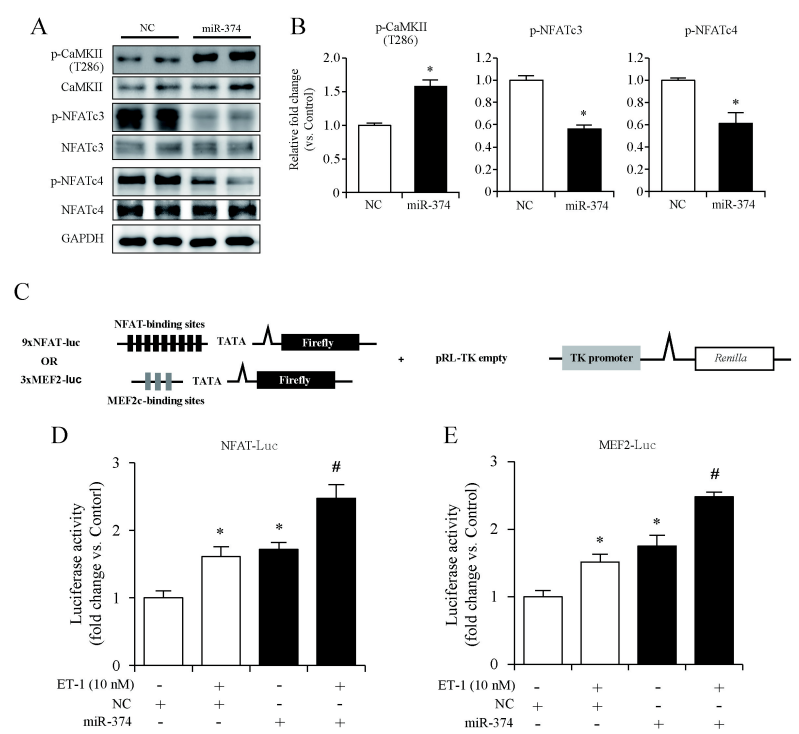

Fig. 3. miR-374 activated prohypertrophic signaling pathways. (A) NRVMs were transfected with NC or miR-374 (20 nM each) as described in the Materials and Methods. The cells were harvested $72 \mathrm{~h}$ after transfection. The levels of p-CaMKII, p-NFATc3, and p-NFATc4 were examined by western blot analysis. (B) The expression levels of the proteins shown in Fig. 3A were estimated by measuring band densities using $\mathrm{NIH}$ Image software. The total protein expression levels of CaMKII, NFATc3, and NFATc4 were used for normalization. GAPDH was used as a loading control. (C) Firefly luciferase reporter constructs containing nine tandem repeats of the consensus binding site for NFAT $(9 \times$ NFAT-luc) and three tandem repeats of the binding site for MEF2 $(3 \times$ MEF2-luc) are shown. pRL-TK was used for normalization. (D) Luciferase activity in NC or miR-374-overexpressing cardiomyocytes cotransfected with pRL-TK and $9 \times$ NFAT-luc or $3 \times$ MEF2-luc in response to ET-1. Significance was measured by two-way ANOVA. $* P<$ 0.05 compared with the NC control. ${ }^{\#} \mathrm{P}<0.05$ compared with the $\operatorname{miR}-374$ control.

transfection with $20 \mathrm{nM}$ miRNA-374 (Fig. 2B and Fig. S2A).

The cell surface area and expression of $B N P$, a hypertrophic marker gene, were increased by VEGFR1 knockdown and were further increased by treatment with ET-1 (Fig. 4). The expression levels of $\mathrm{p}-\mathrm{NFATc} 4$ and the modulatory calcineurin interacting protein 1.4 (MCIP1.4), a molecule downstream of NFAT, were significantly increased by VEGFR1 knockdown (Fig. 4D).

Collectively, these data demonstrated that knockdown of VEGFR1 alone could induce myocardial hypertrophy, suggesting that VEGFR1 indeed mediates the prohypertrophic role of miR-374.

\section{DISCUSSION}

The novel findings of this study are as follows: 1) $m i R-374$ played a prohypertrophic role in the heart (Fig. 1), 2) miR-374 targeted VEGFR1 and PKG-1 (Fig. 2), 3) the prohypertrophic 

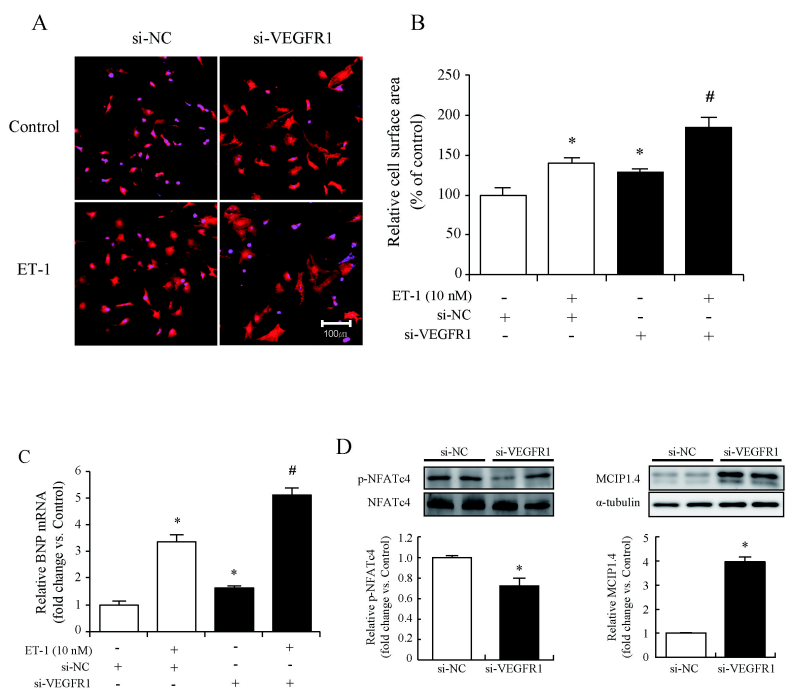

Fig. 4. Inhibition of VEGFR1 by siRNA induced cardiomyocyte hypertrophy. (A) Representative photographs of NRVMs transfected with either $50 \mathrm{nM}$ si-NC or $50 \mathrm{nM}$ si-VEGFR1 in the presence or absence of ET-1. Sarcomeric organization of the cardiomyocytes was visualized by staining with an anti- $\alpha$-actinin antibody. (B) Cell surface areas were measured by using NIH Image software ( $\mathrm{n}=$ 100 cells). Scale bar, $100 \mu \mathrm{m}$. Significance was measured by two-way ANOVA. ${ }^{*} \mathrm{P}<0.05$ compared with the si-NC control. ${ }^{\#} \mathrm{P}<0.05$ compared with the si-VEGFR1 control. (C) qRT-PCR analysis of BNP expression in 50 nM si-NC or si-VEGFR1transfected/ET-1-treated NRVMs. The qRT-PCR analysis was performed in triplicate with three independent samples. Data are expressed as the fold change \pm SD versus the control group. Significance was measured by two-way ANOVA. ${ }^{*} \mathrm{P}<0.05$ compared with the si-NC control. " $\mathrm{P}<0.05$ compared with the si-VEGFR1 control. (D) p-NFATc4 and MCIP1.4 protein levels were measured in NRVMs transfected with si-NC or si-VEGFR1 (50 $\mathrm{nM}$ each) using western blot analysis. The protein expression level was estimated by measuring band densities using $\mathrm{NIH}$ ImageJ software. NFATc4 and $\alpha$-Tubulin were used as a loading control. si-NC, negative control siRNA; si-VEGFR1, siRNA for VEGFR1.

role of miR-374 could be explained by inhibition of the GPCR-associated prohypertrophic $\mathrm{Ca}^{2+}$-signaling pathway (Fig. 3), and 4) siRNA-mediated knockdown of VEGFR1 or PKG-1 by itself could induce myocardial hypertrophy (Fig. 4 and Fig. S2). Taken together, our findings provided evidence of a new type of regulatory mechanism for the development of cardiac hypertrophy (Fig. S1).

In the past decade, several studies have revealed that miRNAs are highly involved in cardiovascular symptoms and diseases. miRNAs can be either prohypertrophic or antihypertrophic regulators (11). For example, overexpression of miR-185, a negative regulator of cardiac hypertrophy, reduces ET-1-induced hypertrophic responses, such as enlarged cell surface area and hypertrophic marker gene expression (12), whereas overexpression of $m i R-19 \mathrm{a} / \mathrm{b}$, a positive regulator of cardiac hypertrophy, increases ET-1-induced hypertrophic responses (16) similar to the effects of miR-374 shown in this study. However, the functional roles of the different types of miRNAs expressed during or at the onset of cardiac diseases have not been clearly documented.

The data shown in Fig. S3 suggested that the expression level of miR-374 was decreased at the onset of the hypertrophic process during trans-aortic constriction (TAC), possibly due to its homeostatic counter-balancing role (Fig. 1). Conversely, the expression level of VEGFR1, a target of miR-374, was increased in ET-1-treated cardiomyocytes (Fig. $\mathrm{S} 2 \mathrm{~A}$ ), possibly due to the downregulation of miR-374 during the onset of myocardial hypertrophy.

VEGF is an endothelial cell mitogen that has essential roles in both vasculogenesis and angiogenesis (17). The functional role of VEGF is mediated by two main tyrosine receptor kinases, VEGFR1 (Flt-1) and VEGFR2 (KDR) (18). In cardiomyocytes, VEGFR2 is involved in the hypertrophy signaling pathway, whereas VEGFR1 is involved in regression signaling by activating PKG-1 $(6,19)$.

Activation of PKG-1 can lead to regression of cardiac hypertrophy by directly inhibiting several $\mathrm{Ca}^{2+}$-dependent regulatory proteins (20). Activation of PKG-1 can result in the phosphorylation of vasodilator-stimulated phosphoprotein (VASP) and attenuate cellular $\mathrm{Ca}^{2+}$ entry (21). The regulator of G-protein signaling 2 (RGS2) is another target of PKG-1. PKG-1 activates RGS2 by phosphorylation and suppresses the activation of the Gq-coupled $\mathrm{Ca}^{2+}$ signaling pathway (22). A recent study provided evidence that PKG-1 can inhibit the NFAT pathway and modulate cell hypertrophy by regulating L-type $\mathrm{Ca}^{2+}$ channels (23). miR-374 may inhibit the regression pathway by targeting VEGFR1 and PKG-1, resulting in promotion of myocardial hypertrophy (Fig. S1).

In the present study, we directly investigated the role of PKG-1 and VEGFR1 in cardiac hypertrophy by using siRNAs specific for PKG-1 and VEGFR1. The results showed that reduction of PKG-1 mRNA levels (32\%) increased the expression levels of the hypertrophic markers ANF and BNP, but decreased the expression levels of p-NFATc4 (Fig. S2 C-E), further confirming that downregulation of PKG-1 was indeed important for myocardial hypertrophy. Similarly, inhibition of VEGFR1 induced cardiomyocyte hypertrophy in both the control and ET-1 treated groups (Fig. 4). However, Zhou et al. (6) previously showed that knockdown of VEGFR1 leads to pro-hypertrophic effects only in the presences of $\mathrm{Cu}$. The discrepancy could be due to the different methods used to measure cardiomyocyte hypertrophy. That is, Zhou et al. (6) used trypsinized floating NRVMs for their flow cytometry experiments, whereas our group used plate-attached NRVMs to perform immunocytochemistry.

In conclusion, the present study demonstrated a new type of pro-hypertrophic regulation by miR-374 through targeting VEGFR1 and PKG-1. Therefore, the prohypertrophic role of $m i R-374$ is likely to be mediated by inhibition of the cardiac hypertrophy regression pathway. Thus, this study may contri- 
bute to our understanding of the pathogenesis of cardiac diseases associated with cardiac hypertrophy.

\section{MATERIALS AND METHODS}

\section{Animal models}

All animal experiments in this study were approved by the Animal Care Committee of Gwangju Institute of Science and Technology (approval number: GIST 2012-15), and were performed according to the guidelines of the GIST Institution Animal Care and Use Committee (IACUC) the NIH principles for the Care and Use of Laboratory Animals. All efforts were made to minimize animal suffering.

\section{Cell culture}

NRVMs were obtained from 1-2-days-old Sprague-Dawley rats, as described previously (24). Briefly, rat ventricular tissue was enzymatically dissociated using $1 \mathrm{mg} / \mathrm{ml}$ collagenase solution (Collagenase type II; Gibco, Grand Island, NY, USA), and the resulting cell suspension was enriched for cardiomyocytes using Percoll (GE Healthcare, Piscataway, NJ, USA) step gradients. Isolated cardiomyocytes were seeded onto either gelatin-coated culture dishes or coverslips and then cultured in cardiomyocyte culture medium: Dulbecco's modified Eagle's medium (DMEM) supplemented with 10\% fetal bovine serum, $1 \%$ antibiotics cocktail, $2 \mathrm{mM}$ L-glutamine, and $100 \mu \mathrm{M}$ 5-bromodeoxyuridine (BrdU, Gibco-BRL) at $37^{\circ} \mathrm{C}$ in an atmosphere containing $5 \% \mathrm{CO}_{2}$.

\section{miRNA transfection and hypertrophic stimulation}

miRIDIAN miRNA mimic rno-miR-374, and miRIDIAN miRNA mimic negative control \#1 (NC) were purchased from Dharmacon (GE Healthcare). NRVMs were cultured in serum-free medium for at least $24 \mathrm{~h}$, and each miRNA (20 nM) was then transfected into NRVMs using DharmaFECT-1 according to the manufacturer's protocol (Dharmacon). After $48 \mathrm{~h}$, to induce hypertrophy, cardiomyocytes were treated with a hypertrophic agonist (10 nM ET-1) for $24 \mathrm{~h}$.

\section{Immunostaining and cell size measurement}

NRVMs grown on gelatin-coated cover slips were fixed with $4 \%$ paraformaldehyde for $10 \mathrm{~min}$, permeabilized with $0.5 \%$ Triton X-100 in PBS for 10 min, and blocked by incubation in $5 \%$ bovine serum albumin (BSA) solution for $1 \mathrm{~h}$ at room temperature $\left(25^{\circ} \mathrm{C}\right)$. The cells were incubated with anti- $\alpha$ actinin antibodies (Sigma-Aldrich, St. Louis, MO, USA) diluted 1:200 at $4^{\circ} \mathrm{C}$ overnight. As a secondary antibody, we used Alexa Fluor 594-coupled anti-mouse antibodies (Invitrogen, Carlsbad, CA, USA) at a dilution of 1:200 and incubated the cells for $1 \mathrm{~h}$ at room temperature $\left(25^{\circ} \mathrm{C}\right)$. Immunofluorescence was analyzed under a microscope equipped with a $40 \times$ objective lens and filters for epifluorescence (Olympus). Cell surface areas were measured using $\mathrm{NIH}$ ImageJ software.

\section{Quantitative real-time PCR (qRT-PCR)}

TRI reagent (Sigma-Aldrich) was used for total RNA extraction from NRVMs. To assess cardiac hypertrophic marker gene expression levels, reverse-transcriptase reactions were performed using PrimeScript RT Master Mix (Takara Bio, Otsu, Japan) with oligo-dT priming. qRT-PCR was performed using a Step One Plus real time PCR system (Applied Biosystems, Foster City, CA, USA) with SYBR Green (Kapa Biosystems, Boston, MA, USA) as a fluorescent dye. The primer sequences are shown in Supplementary Table 1.

\section{Silencing of protein expression by siRNA transfection}

NRVMs were transfected with $50 \mathrm{nM}$ siRNAs using DharmaFECT-1 reagent according to the manufacturer's instructions. siRNAs for VEGFR1 and PKG-1 were purchased from Dharmacon (On-TARGETplus; Dharmacon).

\section{Western blot analysis}

Protein samples were prepared from NRVMs using $1 \%$ sodium dodecyl sulfate (SDS) lysis buffer supplemented with protease inhibitor cocktail (Roche Diagnostics, Mannheim, Germany) and phosphatase inhibitor cocktail (Roche Diagnostics). Protein samples were separated by SDS-PAGE and transferred to polyvinylidene difluoride (PVDF) membranes (Millipore, Billerica, MA, USA), followed by blocking with $5 \%$ BSA (Sigma-Aldrich) in TBST $(0.1 \%$ Tween-20 in Tris-buffered saline: $137 \mathrm{mM} \mathrm{NaCl}$ and $20 \mathrm{mM}$ Tris- $\mathrm{HCl}, \mathrm{pH}$ 7.4) for $1 \mathrm{~h}$ at room temperature. For NFAT, $8 \%$ skim milk (Difco, Detroit, MI, USA) in TBST was used for blocking. Membranes were then incubated overnight at $4^{\circ} \mathrm{C}$ with the following primary antibodies: anti-VEGFR1, anti-PKG-1, anti-phospho (p)-CaMKII (Thr286) (Cell Signaling Technology, MA, USA), anti-p-NFATc3, anti-p-NFATc4, anti- $\alpha$-tubulin (Santa Cruz Biotechnology, Santa Cruz, CA, USA). Next, the membranes were incubated with the appropriate horseradish peroxidase (HRP)-conjugated secondary antibodies (AbFrontier, Seoul, Korea) at room temperature for $1 \mathrm{~h}$, and luminescence was detected using an ImageQuant LAS 4000 mini (GE Healthcare) and a SuperSignal West Pico Chemiluminescence Kit (Thermo Fisher Scientific, Waltham, MA, USA). The intensity of each protein band was quantified by NIH ImageJ software.

\section{Luciferase reporter assay}

For target gene validation, we constructed a reporter vector containing the 3'UTR of the target gene. A luciferase assay was performed using the Dual-GLO Luciferase Assay System (Promega, Madison, WI, USA) according to the manufacturer's instructions. Briefly, we seeded human embryonic kidney (HEK-293) cells into 12-well plates, and cotransfected the cells with $1.5 \mu \mathrm{g}$ of pmirGLO plasmid and $20 \mathrm{nM}$ or $50 \mathrm{nM}$ miRNA mimics. After further cultivation for $72 \mathrm{~h}$, cells were harvested and the luciferase activities were measured using a Victor3 luminometer (PerkinElmer Life and Analytical Sciences, Waltham, MA, USA). Relative luciferase activities were calcu- 
lated as the ratio of firefly to Renilla luciferase activity. For measuring NFAT and MEF2 transcription factor activity, luciferase assays were performed as previously described with minor modifications (16). Briefly, the $9 \times$ NFAT- or $3 \times$ MEF2luciferase reporter plasmid with pRL-TK containing the Renilla luciferase gene was cotransfected into NRVMs with $20 \mathrm{nM}$ miRNA mimics. After $24 \mathrm{~h}$, to induce hypertrophy, cells were stimulated with $10 \mathrm{nM} \mathrm{ET-1}$ for $24 \mathrm{~h}$. The luciferase reporter plasmid driven by $9 \times$ NFAT binding sites was kindly provided by Dr. Jeffery D. Molkentin (University of Cincinnati).

\section{Statistical analysis}

All data are reported as the mean \pm standard deviation (SD). Statistical significance was analyzed using a two-way analysis of variance (ANOVA) with a Bonferroni post-hoc analysis for multiple comparisons with Prism 6.0 (GraphPad Software, Inc, La Jolla, CA, USA). In all cases, differences with $P$ values of less than 0.05 were considered statistically significant.

\section{ACKNOWLEDGEMENTS}

This work was supported by the 2016 GIST Research Institute (GRI) Grant, and the grant from the National Research Foundation of Korea (NRF) funded by the Korean government, the Ministry of Science, ICT, \& Future Planning (NRF-2013M 3A9A7046297).

\section{CONFLICTS OF INTEREST}

Authors have no conflicting financial interests.

\section{REFERENCES}

1. Frey N and Olson EN (2003) Cardiac hypertrophy: the good, the bad, and the ugly. Annu Rev Physiol 65, 45-79

2. Rockman HA, Koch WJ and Lefkowitz RJ (2002) Seventransmembrane-spanning receptors and heart function. Nature 415, 206-212

3. Heineke J and Molkentin JD (2006) Regulation of cardiac hypertrophy by intracellular signalling pathways. Nat Rev Mol Cell Biol 7, 589-600

4. Ferrara N (1999) Molecular and biological properties of vascular endothelial growth factor. J Mol Med 77, 527-543

5. Zhou Y, Jiang Y and Kang YJ (2008) Copper reverses cardiomyocyte hypertrophy through vascular endothelial growth factor-mediated reduction in the cell size. J Mol Cell Cardiol 45, 106-117

6. Zhou Y, Bourcy K and Kang YJ (2009) Copper-induced regression of cardiomyocyte hypertrophy is associated with enhanced vascular endothelial growth factor receptor-1 signalling pathway. Cardiovasc Res 84, 54-63

7. Olsson AK, Dimberg A, Kreuger J and Claesson-Welsh L (2006) VEGF receptor signalling - in control of vascular function. Nat Rev Mol Cell Biol 7, 359-371
8. Cantley LC (2002) The phosphoinositide 3-kinase pathway. Science 296, 1655-1657

9. Takahashi T, Ueno H and Shibuya M (1999) VEGF activates protein kinase C-dependent, but Ras-independent Raf-MEK-MAP kinase pathway for DNA synthesis in primary endothelial cells. Oncogene 18, 2221-2230

10. Lai EC (2002) Micro RNAs are complementary to 3' UTR sequence motifs that mediate negative post-transcriptional regulation. Nat Genet 30, 363-364

11. Wang J, Liew OW, Richards AM and Chen YT (2016) Overview of MicroRNAs in Cardiac Hypertrophy, Fibrosis, and Apoptosis. Int J Mol Sci 17, 749-769

12. Kim JO, Song DW, Kwon EJ et al (2015) miR-185 plays an anti-hypertrophic role in the heart via multiple targets in the calcium-signaling pathways. PLoS One 10, e0122509

13. Small EM and Olson EN (2011) Pervasive roles of microRNAs in cardiovascular biology. Nature 469, 336-342

14. Ganesan J, Ramanujam D, Sassi Y et al (2013) MiR-378 controls cardiac hypertrophy by combined repression of mitogen-activated protein kinase pathway factors. Circulation 127, 2097-2106

15. Castaldi A, Zaglia T, Di Mauro V et al (2014) MicroRNA133 modulates the $\beta 1$-adrenergic receptor transduction cascade. Circ Res 115, 273-283

16. Song DW, Ryu JY, Kim JO et al (2014) The miR-19a/b family positively regulates cardiomyocyte hypertrophy by targeting atrogin-1 and MuRF-1. Biochem J 457, 151-162

17. Carmeliet P and Collen D (2000) Molecular basis of angiogenesis. Role of VEGF and VE-cadherin. Ann N Y Acad Sci 902, 249-62

18. Neufeld G, Cohen T, Gengrinovitch $S$ and Poltorak Z (1999) Vascular endothelial growth factor (VEGF) and its receptors. FASEB J 13, 9-22

19. Shiojima I, Sato K, Izumiya Y et al (2005) Disruption of coordinated cardiac hypertrophy and angiogenesis contributes to the transition to heart failure. J Clin Invest $115,2108-2118$

20. Gray DB, Polo-Parada L, Pilar GR et al (1999) A nitric oxide/cyclic GMP-dependent protein kinase pathway alters transmitter release and inhibition by somatostatin at a site downstream of calcium entry. J Neurochem 72, 1981-1990

21. O'Neil RG (2007) VASP: a TRPC4-associated phosphoprotein that mediates PKG-induced inhibition of storeoperated calcium influx. Am J Physiol Renal Physiol 293, F1766-7

22. Tang KM, Wang GR, Lu P et al (2003) Regulator of G-protein signaling-2 mediates vascular smooth muscle relaxation and blood pressure. Nat Med 9, 1506-1512

23. Vandael DH, Mahapatra S, Calorio C, Marcantoni A and Carbone E (2012) Cav1.3 and Cav1.2 channels of adrenal chromaffin cells: emerging views on cAMP/cGMP-mediated phosphorylation and role in pacemaking. Biochim Biophys Acta 1828, 1608-1618

24. Jeong $\mathrm{MH}$, Kim SJ, Kang $\mathrm{H}$ et al (2015) Cucurbitacin I Attenuates Cardiomyocyte Hypertrophy via Inhibition of Connective Tissue Growth Factor (CCN2) and TGF$\beta /$ Smads Signalings. PLoS One 10, e0136236 